\title{
Dynamic and Decentralized Trust Management for the Internet of Things (IoT) paradigm
}

\author{
Kazi Masum Sadique ${ }^{[0000-0002-9423-6270]}$, Rahim Rahmani ${ }^{[0000-0001-5924-5457]}$ and \\ Paul Johannesson ${ }^{[0000-0002-7416-8725]}$ \\ Department of Computer and Systems Sciences, Stockholm University, Borgarfjordsgatan 8, \\ SE-16407 Kista, Sweden \\ https://dsv.su.se/en/ \\ \{sadique, rahim, pajo\} ddsv.su. se
}

\begin{abstract}
Trust is an invisible behavior of any entity. An entity could be a living being or a cyber-physical system. The Internet of Things (IoT) is a connected network of smart objects or things where trusted relationships are crucial. Trust in an entity can increase or decrease based on different parameters and properties of the specific entity. Trusted relationships can dynamically reach based on contextual data collected over time. The heterogeneous behavior of IoT devices makes trust measurement more difficult. The massive deployment of IoT devices and related innovative IoT applications leads to exploring new trust management frameworks for the IoT paradigm. Emerging IoT applications need to trust entities deployed by third-party providers. Innovative external IoT applications need to be dynamically trusted by the IoT devices and IoT gateways. Dynamic trust achievement is a complex process when an entity is new within the network. In this article, we have defined the trust management for IoT and discussed the need for trusted architecture for dynamic IoT infrastructure, and elaborated the requirements of trust management policies. We have also heightened the need for decentralized architecture for trust management for the Internet of Things (IoT). A new edge-centric multi-agent-based dynamic and decentralized trust management model is proposed and simulated to solve the aforementioned issues. The results of this work are useful for further research in the field of trust management for IoT.
\end{abstract}

Keywords: Trust Management, Internet of Things (IoT), Dynamic Trust, Decentralized Architecture, Properties of Trust.

\section{Introduction}

The recent innovative use cases of the Internet of Things (IoT) required mutual collaboration between IoT devices and gateways. The wireless sensor devices need to transmit data between each other. The machine to machine communication and cooperation increases with wireless sensor networks' vast deployment, which are the core IoT infrastructure component. The IoT devices need to trust each other before sharing data [1]. The wireless sensor devices are mostly resource-constrained devices and 
mostly battery-driven devices with less memory, and computational power [1]. Trust is an invisible property of any entity, which can be measured based on and built upon previous activates of that specific entity. The building of a trusted relationship is possible based on different parameters. Cooperation and noncooperation between various entities are frequent parameters for trust calculation. The probability of trustworthiness of an entity can be measured based on the previous record of collaboration of that specific entity [2]. One another property for calculation of trust is information. A truster can achieve a trust relationship with a trustee based on the information that the truster has about the trustee [3]. Mistrust, untrust, and distrust are negative words of trust. These words are applicable in different situations and based on the available information at a truster about a trustee at a particular time [3]. For example, mistrust can happen in a situation when a truster entity is misleading to trust a trustee entity. In this situation, the trustee may be innocent, but the truster may not trust the trustee due to a lack of proper information.

A sensor node may trust the second node in an IoT communication scenario and send sensitive information for further forwarding to the next entity. But the second node could die due to a power issue. In this situation, the second node was node malicious. Still, the sender node was misguided as it did not have the information about the battery power status of the second wireless sensor device. A truster IoT device can untrust a trustee IoT device due to less knowledge on that specific IoT device. Distrust can happen when a trustee IoT device lacks trust in a trustee IoT device that the second IoT device can perform a particular task. For example, an IoT end device can distrust another IoT end device for making a decision based on individual data it shares with that specific IoT device. The same IoT device can trust an IoT gateway for getting particular tasks done. Because an IoT gateway has more computation power and is connected to a power source, and can perform specific computational functions that may be impossible for an IoT device.

As the IoT paradigm is based on heterogeneous wireless sensor networks, it is not each to have enough information about devices for making a decision on trust and doing cooperation. IoT devices may have mobility capabilities and can be victims of an attack at any time. It is impossible always to trust an IoT device based on static information about the device saved in a particular database table. So, we need to dynamically calculate the trust based on the available information, leading to the requirement for dynamic trust management for the internet of things (IoT). Additionally, achieving dynamic trust is not feasible in a centralized IoT architecture because the IoT devices need to make decisions quickly. Verification of information from a centralized system is not possible as quickly as it is possible to verify from a server near the IoT device. As a result, we need a decentralized architecture for trust management for edge IoT devices and edge IoT Gateways.

The rest of the paper is organized as follows: in section 2, we have the background and motivation. In section 3, we have the related works. Section 4 presents our proposed decentralized trust management model. Section 5 presents simulation results of our proposed model followed by section 6 , the conclusion, and future works. 


\section{Background and Motivation}

There are different ways of trust calculation. Dynamic trust can be calculated based on the information that a truster IoT device knows about a trustee IoT device. An IoT device might be influenced by the information from a neighbor IoT device. Here comes the concept of trust propagation [4]. Trust information coming from another IoT device, could be biased sometimes; in that case, the result of trust calculation could be misleading [4] and can be ended up with distrust or mistrust. Trust propagation happens when social networking [5,6] between entities is established [7]. Trust can be achieved on a trustee entity based on the reputation of that entity and selfassessment by a truster entity [8]. Self-assessment can be performed based on various contextual information of a trustee entity [8]. Trust can be subjective and objective [9]. The objective trust is built based on certain policies or predefined information available about a specific entity. Objective trust is mostly built upon static information. But subjective trust can be achieved based on personal experiences, understanding, and communality [9]. A trusted relationship can change based on the control over a trustee. Suppose a truster has better control over a trustee, the trust index's value increases [9]. There are cases when a user device or a user application needs to trust a certain or a group of IoT devices. This can be calculated based on different criteria like ownership [10,11], control [10,11], heterogeneity, composability, interoperability, compatibility, etc. [11].

Trust aggregation is another term used in the trust management process, where current trust information from neighbors is collected for further processing [12]. Another way of trusting any entity is authentication [13] and verification of the identity of an IoT entity $[14,15,16,19]$. Another way of enhancing trust in IoT is a hardware-based trust mechanism [17]. Suppose we can ensure that the device consists of a trusted execution environment [TEE] $[6,18,19]$. In case of any malicious attack, the trusted execution area of the device is not accessible by the attacker. In that case, we can ensure that the device is trustworthy [17]. Direct interaction, negotiation of trust information, the reputation of a device [20], recommendation by others are the popular measurement standards of trust calculation, which lead to trust propagation $[4,21]$.

A compromised IoT device can introduce a big security hole within the IoT network. In case a compromised IoT device is considered trusted, it increases the risks more [22]. In decentralized communication and decentralized trust management scenario, devices communicate with local authenticators [19, 23]. Because when communication is done between local entities, it is essential to ensure real-time dynamic identity and trust management. We proposed and verified decentralized and dynamic identity management, authentication, and secure communication protocol for edge IoT devices and edge IoT gateways in our earlier research work [19]. During our previous studies, we found that trust management for IoT at the edge/fog layer is more efficient for real-time decision making by the time-critical IoT devices [19, 24]. Influenced by the above, in this paper, we have proposed an edge centric multi-agent based trust negotiation model for IoT. Before describing our proposed model, we have presented different related works in the next section. 


\section{Related Works}

Several recent research works addressed trust calculation, trust management, trust negotiation for IoT. A trust calculation framework (ConTrust) for IoT devices based on current trust and reputation is presented in [25]. The authors proposed a publishersubscriber based model where the election of ConTrust manager between two IoT devices is discussed. The ConTrust manager performs the calculation of trust. The main drawback of this model is the single point of failure. If the ConTrust manager is compromised, it will produce a misleading result and can be harmful. A distributed trust management model for IoT (DTM-IoT) is proposed in [26]. The authors have proposed a clustering-based trust negotiation and trust establishment model. The model consists of three types of nodes. They are the cluster node, master node, and super node [26]. The trust value is shared between these nodes using the Application Programming Interface (API). The work does not contain any simulation or experimental results. A trust management model, with integration to the routing protocol for low-power and lossy networks (RPL), namely the dynamic and comprehensive trust model (DCTM-IoT), is proposed in [27]. The authors discussed the context of trust, belief, uncertainty, criteria, and how trust can be achieved between two different entities. They have also simulated their proposed model using the Cooja simulator in Instant Contiki OS, a lightweight OS platform for wireless sensor networks (WSN) [28]. The authors also discussed three different types of attacks: SYBIL, RANK, BLACKHOLE. Though this paper proposed a fascinating concept, it focuses more on RPL integration with the proposed model. A framework for dynamic trust management using multi-agent systems is proposed in [29]. The proposed framework applies to robotic systems. A protocol for dynamic trust management for IoT is proposed in [30]. The authors considered three trust properties: honesty, community interest, and cooperativeness to calculate trust between components of an IoT paradigm. Indirect recommendations and direct observations are considered for the trust propagation parameters. As our concept is also based on dynamic trust, our idea has small similarities with their idea, but we have proposed dynamic trust with edge-centric decentralized models.

\section{Proposed Solution}

To solve the trust management problem discussed above, we propose a multi-agentbased edge centric model. In our proposed model, IoT devices and edge gateways share their knowledge about their neighbors. The edge gateways work as a hub towards the cloud servers. Trust calculation is also done at the edge gateway within the edge intelligence module (see Fig. 1) because the edge gateway has more computational power compared to an end IoT device. The IoT devices only share and accept information from an authenticated IoT device and from an authenticated edge gateway. Decentralized and distributed authentication should be handled by the edge identity management module (see Fig. 1). We have presented a decentralized and distributed identity management at the edge gateway and at edge/fog layer identity man- 
agement server in [19]. The IoT devices are the lower layer device in the model and only collaborate with the edge gateways. Let us consider a smart city scenario where information about a defective traffic controller needs to be shared with the control center as well as all smart IoT components in that area, which may have an effect due to the fault with that specific traffic light. As soon as a neighbor IoT node detects that a traffic controller is defective, it shares the knowledge to the nearest node, and the nearest node propagates it to the edge gateway. The edge gateway forwards the information to the cloud server, which is connected to the centralized monitoring system and or with a notification system. In the above scenario, the IoT device can directly share the information with the cloud server and not with the nearest neighbors, but that could cause severe problems because it is very important to notify all the devices within the area about the faulty IoT device. The same information can be shared with the IoT devices within that area via a centralized server, but it could be already late because, in a smart city, real-time and quick propagation of information is crucial. Trust on an IoT device or an Edge gateway can be calculated based on honesty, cooperativeness, community interest, and recommendations, similar to those described in [30].

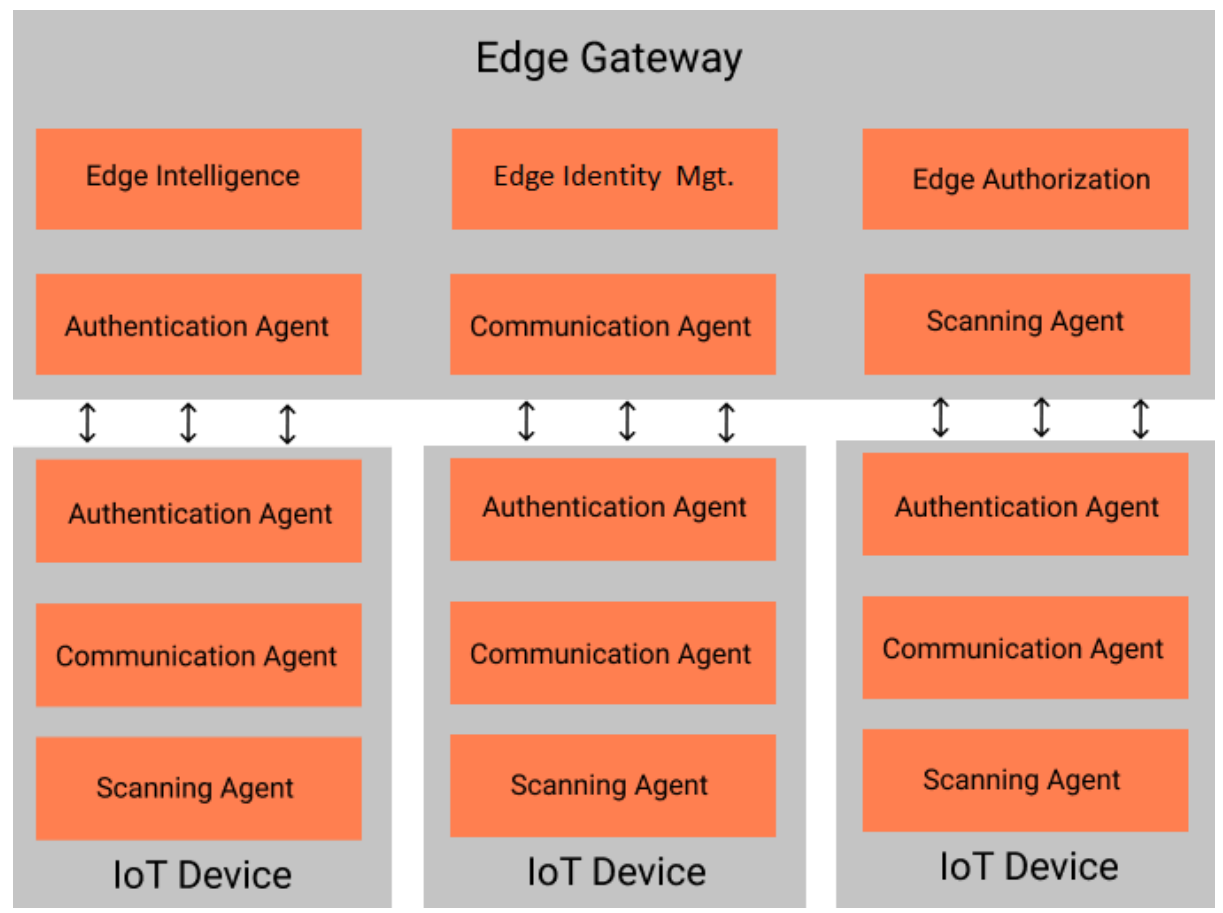

Fig. 1. Proposed edge centric multi-agent based trust management model.

Fig. 1 represents the internal architecture of an edge gateway and IoT devices of our proposed model. The edge gateway consists of six modules: edge intelligence, edge identity management, edge authorization, authentication agent, communication agent, and scanning agent. An IoT device consists of three agent modules: authentica- 
tion agent, communication agent, and scanning agent. The authentication agents in IoT devices and edge gateways are responsible for the authentication of all incoming requests. Authentication and identity management should be performed as per the proposed protocol in our previous work [19]. A scanning agent scans its surrounding for any change or malicious activity. The communication agent shares information with the communication agent on another device. The communication should be secured using the secure communication protocol described in our earlier research [19]. The communication agent and authentication agent should work together to ensure authentic communication. We did not present the cloud server in our proposed model (see Fig. 1) for simplicity, but the cloud layer is also included in our previously proposed decentralized and distributed identity management with secure communication protocol [19]. In the next section, we have presented the simulation results of our proposed dynamic and decentralized trust propagation protocol.

\section{$5 \quad$ Simulation Results}

We have simulated our proposed model using the GAMA platform [30]. GAMA is a popular modeling and simulation platform for agent-based systems. Fig. 2 represents the simulation result of our proposed model.

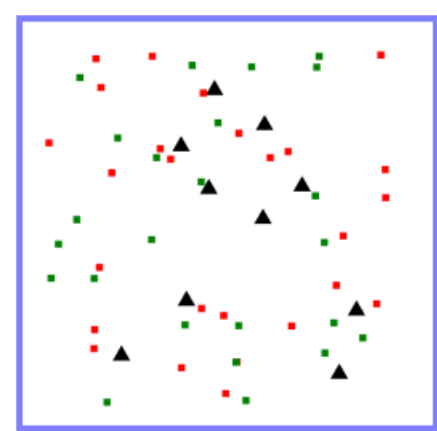

(a)

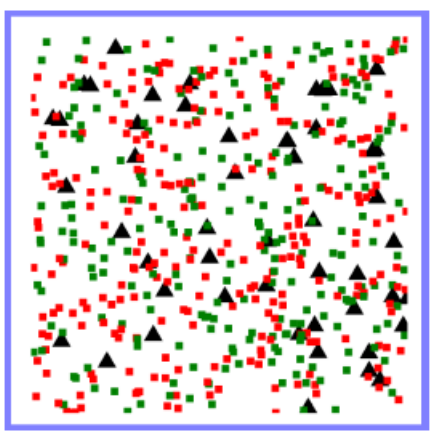

(c)

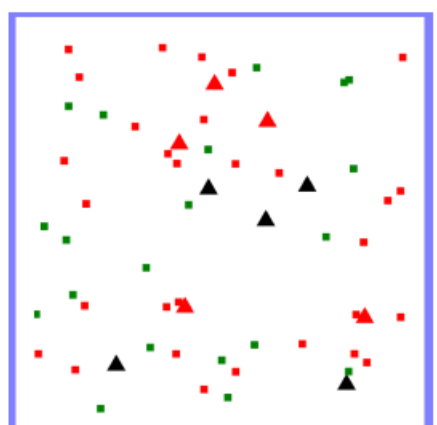

(b)

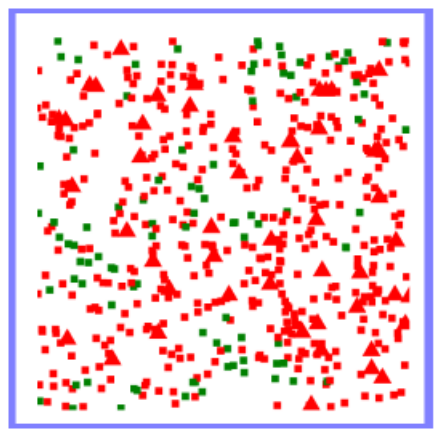

(d)

Fig. 2. Trust propagation between Edge gateway and IoT devices. 
In our simulation model (see Fig. 2), the edge gateways are represented as triangles, and IoT devices are presented as squares. Fig. 2 (a) represents the initial state before simulation for the scenario where ten edge gateways and fifty IoT devices share information between them. The red squares are randomly initially filled with information, and the green square marked IoT devices are not initially filled with information. Fig. 2 (b) represents the result of trust propagation after 30 seconds. Here we can see that several triangles (edge gateways) become red, which got notifications from a nearby IoT device(s). Fig. 2 (c) represents the initial state before simulation for the scenario where fifty edge gateways and five hundred IoT devices share information between them. The red squares are randomly initially filled with information, and the green square marked IoT devices are not initially filled with information. Fig. 2 (d) represents the result of trust propagation after 15 seconds. We can see all the triangles (edge gateways) and the green squares (IoT devices) become red, which got notifications from one of the nearby IoT devices or edge gateway.

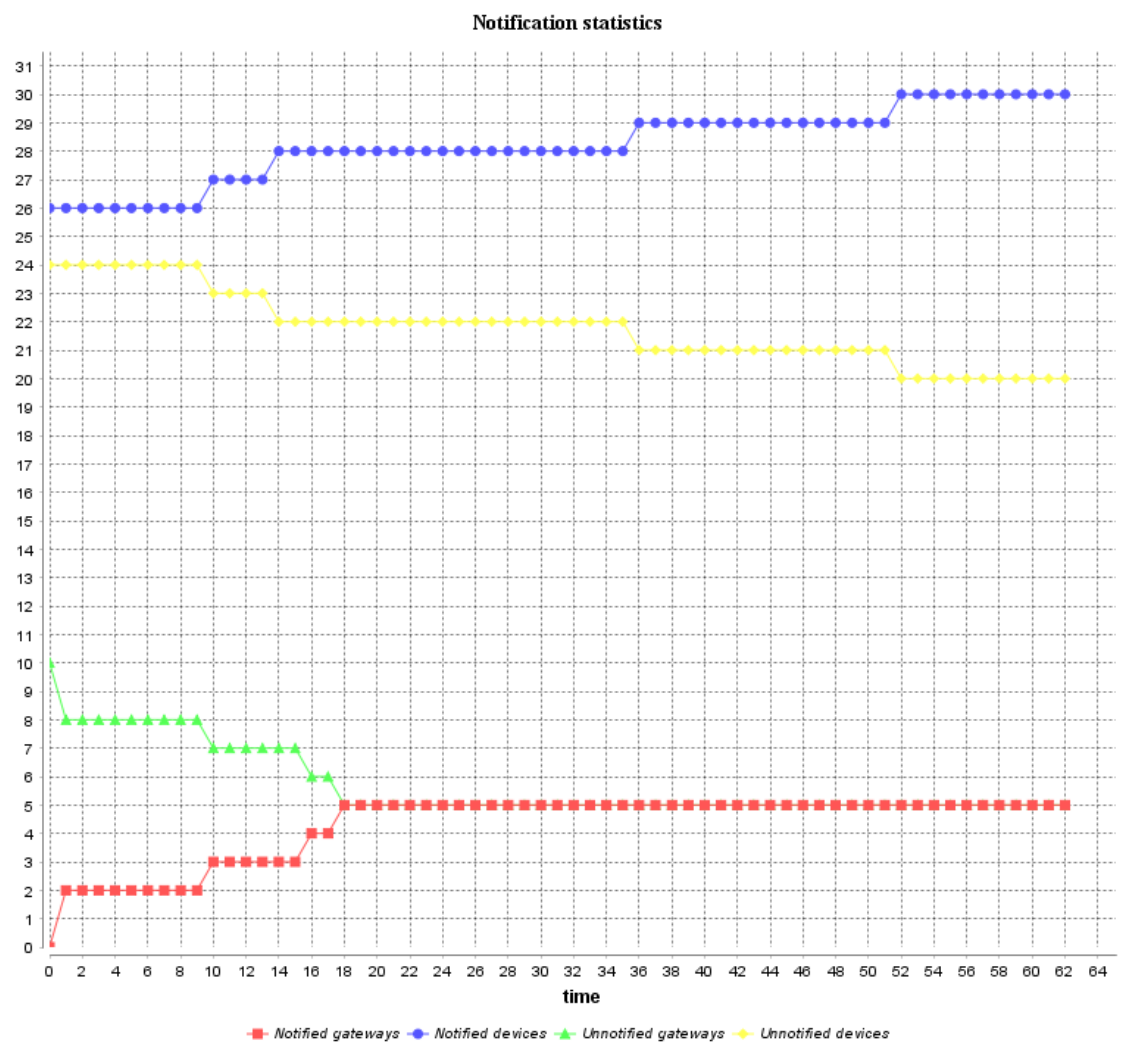

Fig. 3. Trust propagation graph for ten edge gateways and fifty IoT devices.

Fig. 3 represents the graph for ten edge gateways and five hundred IoT devices. Fig. 4 illustrates the graph for fifty edge gateways and five hundred IoT devices. Both 
in Fig. 3 and Fig. 4, the blue line in the graph represents the number of notified IoT devices. The yellow line represents the number of unnotified IoT devices. The red line represents the notified edge gateways, and the green line represents the number of unnotified edge gateways. Fig. 3 and Fig. 4 show that with the increment of notification spread between IoT devices and edge gateways, the number of unnotified devices and edge gateways decreases.

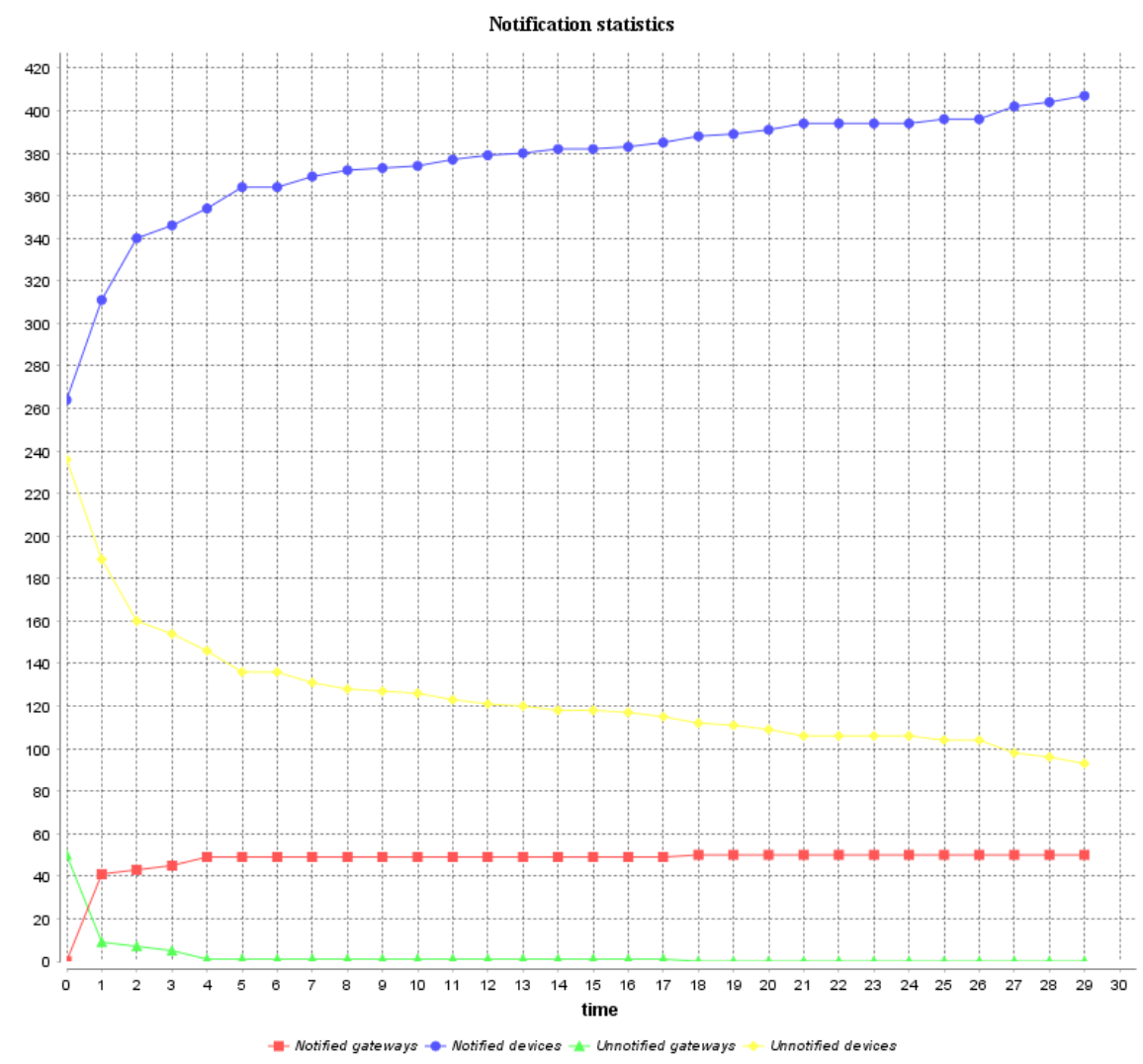

Fig. 4. Trust propagation graph for fifty edge gateways and five hundred IoT devices.

\section{Conclusion and Future Works}

This paper has presented definitions of dynamic trust based on previous works within the trust management domain. We have described the need for dynamic and decentralized trust management for the internet of things (IoT). From the literature review, it has been found that a trusted IoT model is still required for authenticated and trustworthy IoT data processing. We have proposed a novel edge centric multi-agentbased model for trust propagation between IoT devices and edge gateways. The pro- 
posed model has been simulated using the GAMA modeling and simulation platform. We have proposed several agent modules inside the IoT devices and edge gateway. As described earlier, the trust calculation should be performed at the edge intelligence module. In our future research, we will extend the functionalities of this module with details on trust calculation based on different dynamic parameters discussed in this paper.

\section{References}

1. Ben Saied, Y., Olivereau, A., Zeghlache, D., Laurent, M.: Trust management system design for the Internet of Things: A context-aware and multi-service approach. Comput. Secur. 39, 351-365 (2013). https://doi.org/10.1016/j.cose.2013.09.001.

2. Hwang, P., Burgers, W.P.: Properties of trust: An analytical view. Organ. Behav. Hum. Decis. Process. 69, 67-73 (1997). https://doi.org/10.1006/obhd.1996.2673.

3. Marsh, S., Dibben, R., M.: Trust, untrust, distrust, and mistrust - An Exploration of the Dark(er) Side. Int. Conf. Trust Manag. 321-337 (2005). https://doi.org/10.1007/11429760.

4. Guha, R., Raghavan, P., Kumar, R., Tomkins, A.: Propagation of trust and distrust. Thirteen. Int. World Wide Web Conf. Proceedings, WWW2004. 403-412 (2004). https://doi.org/10.1145/988672.988727.

5. Ghavipour, M., Meybodi, M.R.: Trust propagation algorithm based on learning automata for inferring local trust in online social networks. Knowledge-Based Syst. 143, 307-316 (2018). https://doi.org/10.1016/j.knosys.2017.06.034.

6. McGillion, B., Dettenborn, T., Nyman, T., Asokan, N.: Open-TEE - An open virtual trusted execution environment. Proc. - 14th IEEE Int. Conf. Trust. Secur. Priv. Comput. Commun. Trust. 2015. 1, 400-407 (2015). https://doi.org/10.1109/Trustcom.2015.400.

7. Huang, J., Fox, M.S.: An ontology of trust - Formal semantics and transitivity. ACM Int. Conf. Proceeding Ser. 259-270 (2006). https://doi.org/10.1145/1151454.1151499.

8. Huang, J., Nicol, D.M.: Trust mechanisms for cloud computing. J. Cloud Comput. 2, 1-14 (2013). https://doi.org/10.1186/2192-113X-2-9.

9. Tan, Y., Thoen, W.: Toward a Generic Model of Trust for Electronic Commerce. Int. J. Electron. Commer. 5, 61-74 (2000). https://doi.org/10.1080/10864415.2000.11044201.

10. Khan, K.M., Malluhi, Q.: Establishing trust in cloud computing. IT Prof. 12, $20-27$ (2010). https://doi.org/10.1109/MITP.2010.128.

11. Voas, J., Kuhn, R., Laplante, P., Applebaum, S.: Internet of Things (IoT) Trust Concerns. Nist. 50-50 (2018).

12. Guo, J., Chen, I.R., Tsai, J.J.P.: A survey of trust computation models for service management in internet of things systems. Comput. Commun. 97, 1-14 (2017). https://doi.org/10.1016/j.comcom.2016.10.012.

13. Zhan, G., Shi, W., Deng, J.: Design and implementation of TARF: A trust-aware routing framework for WSNs. IEEE Trans. Dependable Secur. Comput. 9, 184-197 (2012). https://doi.org/10.1109/TDSC.2011.58.

14. Sadique, K.M.K.M., Rahmani, R., Johannesson, P., Rahmani, R., Johannesson, P., Rahmani, R., Johannesson, P.: Trust in Internet of Things : An architecture for the future IoT network. 2018 Int. Conf. Innov. Eng. Technol. ICIET 2018. 27-29 (2019). https://doi.org/10.1109/CIET.2018.8660784.

15. Al-Rousan, T., Abuserieah, R., Abualese, H.: Trustworthy framework for cloud of things. ICIT 2017 - 8th Int. Conf. Inf. Technol. Proc. 338-343 (2017). https://doi.org/10.1109/ICITECH.2017.8080023. 
16. Bing, Z., Xin-Xin, M., Zhi-Guang, Q.: Security Architecture on the Trusting Internet of Things. J. Electron. Sci. Technol. 9, 364-367 (2011). https://doi.org/10.3969/j.issn.1674862X.2011.04.014.

17. Sabt, M., Achemlal, M., Bouabdallah, A.: Trusted execution environment: What it is, and what it is not. Proc. - 14th IEEE Int. Conf. Trust. Secur. Priv. Comput. Commun. Trust. 2015. 1, 57-64 (2015). https://doi.org/10.1109/Trustcom.2015.357.

18. Sebastian, D.J., Agrawal, U., Tamimi, A., Hahn, A.: DER-TEE : Secure Distributed Energy Resource Operations Through Trusted Execution Environments. 6, 6476-6486 (2019).

19. Sadique, K.M., Rahmani, R., Johannesson, P.: IMSC-EIoTD: Identity management and secure communication for edge IoT devices. Sensors (Switzerland). 20, 1-38 (2020). https://doi.org/10.3390/s20226546.

20. Pearson, S., Benameur, A.: Privacy, Security and Trust Issues Arising from Cloud Computing. 2010 IEEE Second Int. Conf. Cloud Comput. Technol. Sci. 693-702 (2010). https://doi.org/10.1109/CloudCom.2010.66.

21. Abbadi, I.M., Martin, A.: Trust in the cloud. Inf. Secur. Tech. Rep. 16, 108-114 (2011). https://doi.org/10.1016/j.istr.2011.08.006.

22. Bhattarai, S., Wang, Y.: End-to-End Trust and and Security for Internet of Things Applications. Comput. by IEEE Comput. Soc. 51, 20-27 (2018). https://doi.org/10.1109/MC.2018.2141038.

23. Chainho, P., Drusedow, S., Pereira, R.L., Chaves, R., Santos, N., Haensge, K., Portabales, A.R.: Decentralized Communications: Trustworthy interoperability in peer-to-peer networks. EuCNC 2017 - Eur. Conf. Networks Commun. (2017). https://doi.org/10.1109/EuCNC.2017.7980649.

24. Sadique, K.M., Rahmani, R., Johannesson, P.: Fog Computing for Trust in the Internet of Things (IoT): A Systematic Literature Review. 2020 Int. Conf. Comput. Sci. Eng. Appl. 16 (2020). https://doi.org/10.1109/ICCSEA49143.2020.9132861.

25. Suryani, V., Sulistyo, S., Widyawan, W.: Internet of things (IoT) framework for granting trust among objects. J. Inf. Process. Syst. 13, 1613-1627 (2017). https://doi.org/10.3745/JIPS.03.0088.

26. Alshehri, M.D., Hussain, F., Elkhodr, M., Alsinglawi, B.S.: A Distributed Trust Management Model for the Internet of Things (DTM-IoT). 1-9 (2019). https://doi.org/10.1007/978-3-319-99966-1_1.

27. Hashemi, S.Y., Shams Aliee, F.: Dynamic and comprehensive trust model for IoT and its integration into RPL. J. Supercomput. 75, 3555-3584 (2019). https://doi.org/10.1007/s11227-018-2700-3.

28. Dunkels A, Schmidt O, Finne N, Eriksson J, sterlind F, Tsiftes N, Durvy M (2011) The Contiki OS: the operating system for the Internet of Things. http://www.contiki-os.org.

29. Zikratov, I., Maslennikov, O., Lebedev, I., Ometov, A., Andreev, S.: Dynamic trust management framework for robotic multi-agent systems. Lect. Notes Comput. Sci. (including Subser. Lect. Notes Artif. Intell. Lect. Notes Bioinformatics). 9870 LNCS, 339-348 (2016). https://doi.org/10.1007/978-3-319-46301-8_28.

30. Bao, F., Chen, I.R.: Dynamic trust management for internet of things applications. SelfIoT'12 - Proc. 2012 Int. Work. Self-Aware Internet Things, Co-located with ICAC'12. 1-6 (2012). https://doi.org/10.1145/2378023.2378025.

31. Drogoul, A., Amouroux, E., Caillou, P., Gaudou, B., Grignard, A., Marilleau, N., ... \& Zucker, J. D. (2013, May). Gama: multi-level and complex environment for agent-based models and simulations. In 12th International Conference on Autonomous agents and multi-agent systems (pp. 2-p). Ifaamas. 